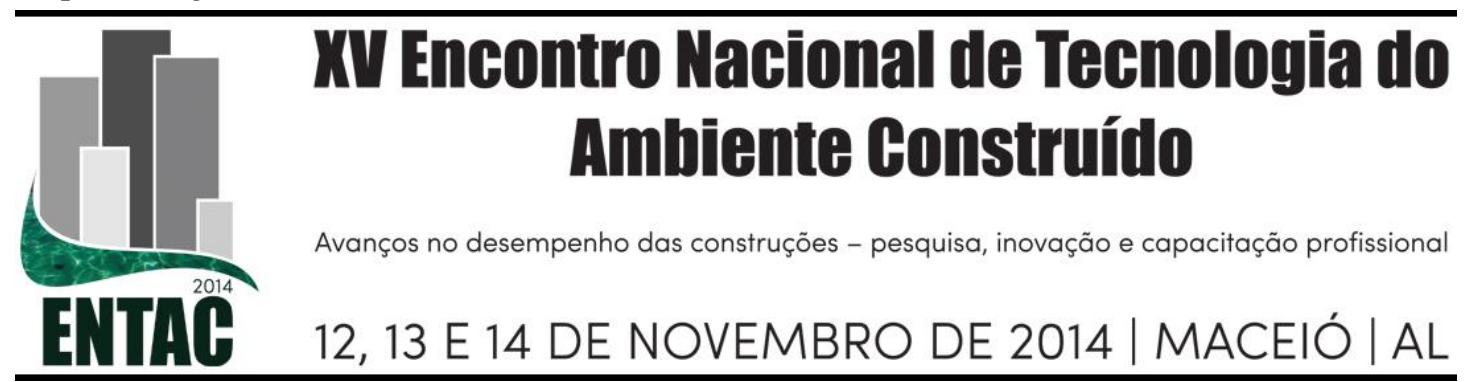

\title{
ANÁLISE DE VARIÂNCIA EM SIMULAÇÃO COMPUTACIONAL DO DESEMPENHO TÉRMICO DE HABITAÇÕES UNIFAMILIARES
}

\author{
SILVA, Arthur Santos (1); ALMEIDA, Laiane Susan Silva (2); SCHAEFER, Aline \\ (3); BALVEDI, Bruna (4); GHISI, Enedir (5) \\ Universidade Federal de Santa Catarina, Departamento de Engenharia Civil, Laboratório de Eficiência \\ Energética em Edificações. (1) arthurssilva07@gmail.com, (2) lai_almeida@hotmail.com, \\ (3) ali_ufsc@yahoo.com, (4) brunabalvedi@gmail.com, (5) enedir@labeee.ufsc.br
}

\begin{abstract}
RESUMO
O objetivo deste artigo é aplicar a "análise de variância" em um experimento de simulação computacional com o intuito de determinar parâmetros mais influentes no desempenho térmico de habitações unifamiliares. Elaborou-se um projeto de experimento para os parâmetros termofísicos de uma habitação unifamiliar para o clima de Campo Grande-MS, e foram realizadas simulações computacionais com o programa EnergyPlus. Nove parâmetros da envoltória da habitação foram variados em dois níveis, totalizando 512 simulações. A análise de variância foi utilizada para explicar a influência de cada parâmetro no desempenho térmico da habitação, o qual foi representado pelos graus-hora de aquecimento e resfriamento. Constatou-se que os parâmetros mais importantes no desempenho para o resfriamento foram a absortância solar e a transmitância térmica da cobertura, e para o aquecimento foram a transmitância térmica das paredes externas e a emissividade da cobertura. Os graus-hora de resfriamento, no caso deste clima, é mais representativo do que os graus-hora de aquecimento; os quais variaram de 84 a $21136^{\circ} \mathrm{Ch}$. Sendo assim, os parâmetros mais influentes no resfriamento podem ter seus valores dimensionados adequadamente de forma a aprimorar o desempenho. Conclui-se que o método da análise de variância é viável para fins de simulação computacional, e tem a capacidade de mostrar com grande precisão estatística, os parâmetros mais influentes no dado de saída analisado. No entanto, o método é limitado pelo número de simulações, o qual cresce exponencialmente com o aumento do número de parâmetros e níveis de variação considerados no experimento, gerando maior esforço computacional e tempo de processamento.
\end{abstract}

Palavras-chave: Análise de variância, Desempenho térmico, Simulação computacional.

\begin{abstract}
The objective of this paper is to promote the "analysis of variance" in a computer simulation experiment regarding the determination of influential parameters on the thermal performance of single-family houses. A design of experiment was elaborated for the thermophysical parameters of a single-family house in the climate of Campo Grande-MS, and computer simulation of the thermal performance using EnergyPlus was performed. Nine parameters related to the envelope of the house were varied on two levels, reaching 512 simulation runs. The analysis of variance was used to explain the influence of each parameter on the thermal performance of the house, which was represented by degree-hours of heating and cooling. One can find that the most important parameters for cooling performance were the solar absorptance and thermal transmittance of the roof, and for the heating performance were the thermal transmittance of the external walls and the roof emissivity. The degree-hour for cooling, in the case of this climate, is more representative than the degree-hour for heating; which ranged from $84-21136^{\circ} \mathrm{Ch}$. One can conclude that the method of analysis of variance is feasible for the purposes of computer simulation, and has the ability to show, with great statistical accuracy, the most influential parameters in the analysed output. However, the method is limited by the number of simulation, which grows exponentially as the number of parameters and variation levels considered in the experiment increase, demanding greater computational effort and processing time.
\end{abstract}




\section{INTRODUÇÃ̃o}

O estudo do desempenho térmico de edificações é um tema de pesquisa recorrente internacionalmente. Nesta área de pesquisa é comum se encontrar trabalhos que tratam de simulação computacional em regime transiente, que analisam determinadas variáveis da edificação considerando o balanço térmico dos elementos envolvidos e os sistemas integrados à edificação (e.g. MARA; TARANTOLA, 2008; HOPFE; HENSEN, 2011; TIAN, 2013). Análises de variáveis que influenciam em determinado comportamento da edificação são comuns, tais como na temperatura interna, nas taxas de ventilação, no consumo de energia ou no desempenho térmico. Estatisticamente, esse tipo de análise de influência corresponde à análise de sensibilidade.

Grande parte das publicações na área de engenharia não utilizam métodos complexos para se analisar a sensibilidade, tratando de utilizar métodos mais simples denominados métodos por triagem (SALTELLI; TARANTOLA; CAMPOLONGO, 2000). Esses métodos são planejados de tal forma que, quando um parâmetro é variado em determinados níveis, os demais parâmetros são mantidos em um nível fixo. É uma forma de análise interessante mas que não possibilita a obtenção de muita informação a respeito da influência de parâmetros, como as interações de segunda ordem.

A análise de variância, por sua vez, é uma forma de determinar a sensibilidade de parâmetros em modelos computacionais, considerando combinações fatoriais completas. Por esse motivo, essa análise possibilita a determinação de parâmetros ou mesmo grupos de parâmetros mais influentes, além de efeitos de segunda ordem (SANCHEZ et al., 2014). Também possibilita selecionar valores mais apropriados para cada um deles dependendo do intuito do experimento.

Alguns estudos podem ser citados, como Chlela et al. (2009) que propuseram um método para se projetar edificações de baixo consumo de energia por meio de simulações paramétricas utilizando a ferramenta estatística de "projeto de experimento". Mechri, Capozzoli e Corrado (2010) analisaram a sensibilidade de parâmetros por meio da análise de variância com a técnica de "amplitude de sensibilidade de Fourier". Os casos simulados foram desenvolvidos com o método da norma internacional ISO 13790 (2008) em regime quase permanente. Da mesma forma, Brohus et al. (2009) analisaram a sensibilidade de parâmetros em uma habitação unifamiliar, de forma a reduzir o número de parâmetros com pré-processamentos.

Mais aplicações dessas técnicas estatísticas precisam ser conduzidas de forma a comprovar sua eficácia em encontrar soluções para o desempenho térmico de edificações, ou mesmo para entender os fenômenos envolvidos na simulação e auxiliar como ferramenta útil em linhas de pesquisa.

O objetivo do trabalho é, portanto, aplicar a análise de variância no desempenho térmico de uma habitação unifamiliar obtido por meio de simulação computacional, decorrente da variação das propriedades termofísicas da envoltória por meio de projeto de experimento.

\section{MÉTODO}

O método divide-se em seis etapas: (1) definição das condições de controle do experimento, (2) configurações padrão das simulações computacionais, (3) projeto de experimento e análise de variância, (4) variáveis dependentes do experimento, (5) experimento computacional, (6) tratamento de dados. 


\subsection{Condições de controle do experimento}

Uma das condições de controle do experimento é a definição do clima. O clima escolhido foi o de Campo Grande-MS. A Figura 1 apresenta algumas variáveis climáticas de Campo Grande, como a temperatura de bulbo seco e úmido, irradiação solar direta e difusa, e umidade relativa. São mostrados dados horários de um dia médio de cada mês, ao longo dos doze meses do ano. A cidade de Campo Grande está localizada na latitude de $20,47^{\circ}$ Sul, longitude de $54,67^{\circ}$ Oeste, e altitude de $556 \mathrm{~m}$.

Os dados horários foram obtidos do arquivo climático SWERA (Solar and Wind Energy Resource Assessment) em formato TMY (Typical Meteorological Year) (DOE, 2014a). O clima é classificado como tropical com estação seca.

A Figura 2 mostra a planta baixa e um croqui isométrico do modelo base da habitação, cujos ambientes analisados foram a sala/cozinha e os três quartos. A orientação solar foi considerada fixa para esse experimento (ver Figura 2).

\section{Figura 1 - Variáveis climáticas de Campo Grande-MS ao longo do ano.}

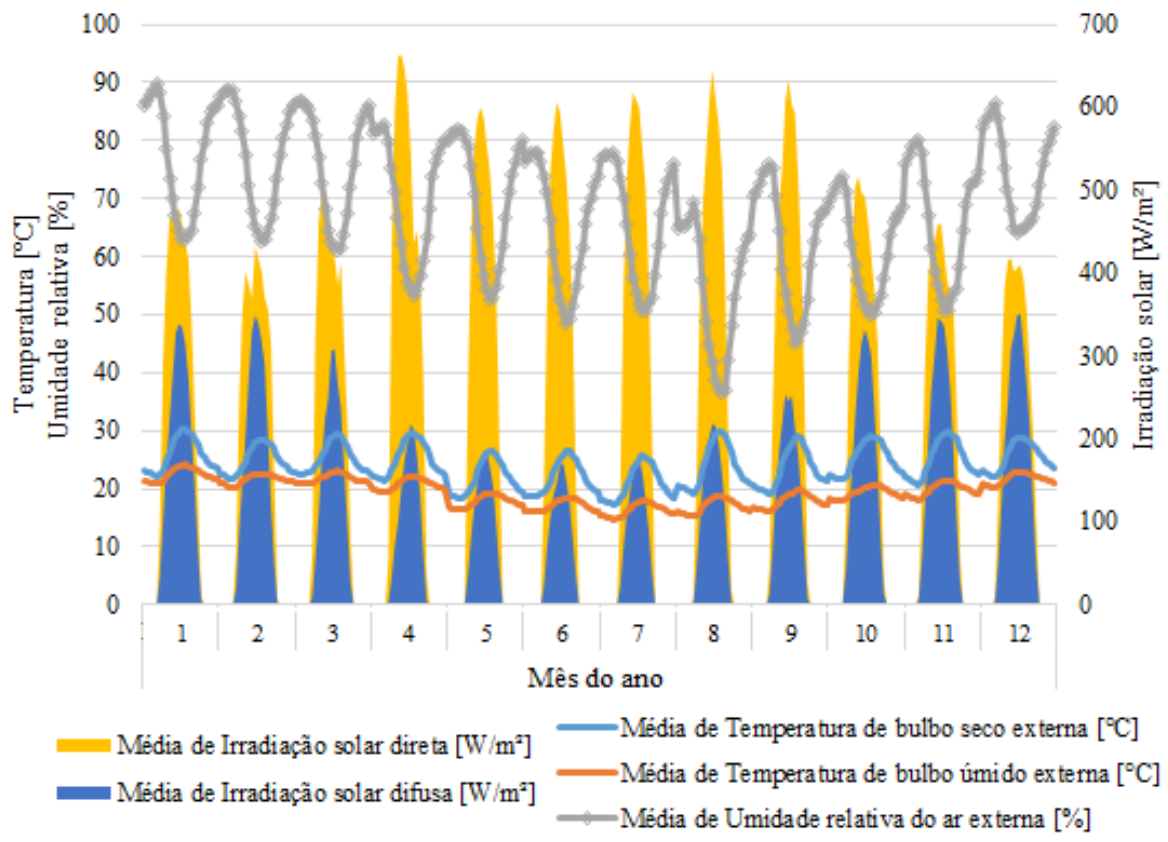

Fonte: Arquivo climático SWERA TMY (DOE, 2014a).

Figura 2 - Modelo base da edificação.

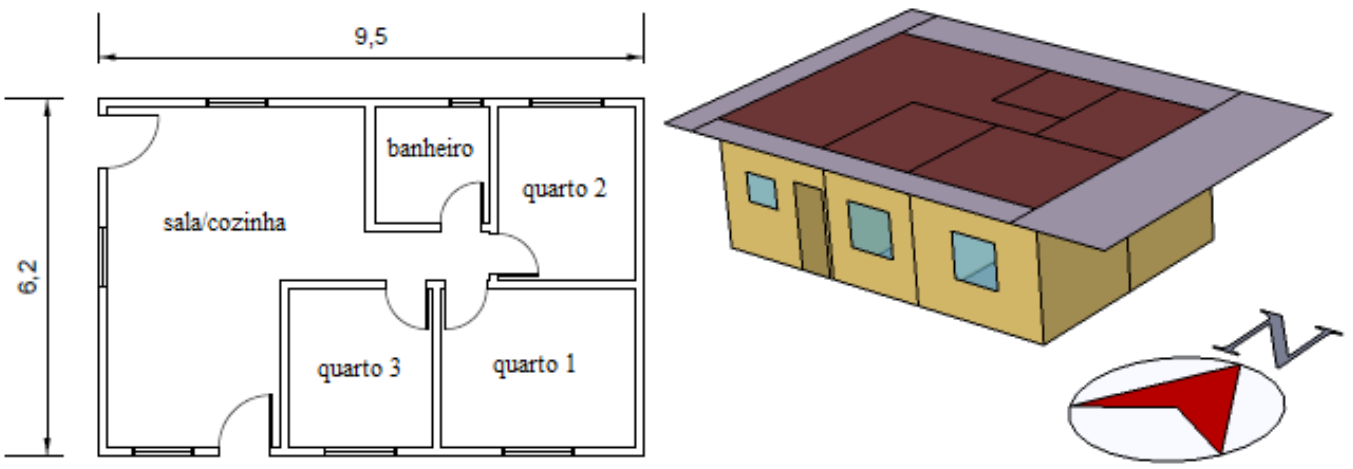




\subsection{Configurações das simulações computacionais}

O experimento conduzido neste trabalho foi realizado por simulação computacional com o programa EnergyPlus versão 8.1 (DOE, 2014b).

As rotinas de ocupação, de uso da iluminação e equipamentos foram adotadas do Regulamento Técnico da Qualidade para o Nível de Eficiência Energética de Edificações Residenciais - RTQ-R (INMETRO, 2012). A Figura 3 mostra a rotina de uso da ocupação e a Figura 4 mostra a rotina de uso da iluminação. Foi fixado o número de ocupantes nos ambientes, sendo dois por dormitório, totalizando seis ocupantes na edificação. A densidade de potência instalada com iluminação foi de $6 \mathrm{~W} / \mathrm{m}^{2}$ na sala e $5 \mathrm{~W} / \mathrm{m}^{2}$ nos dormitórios. A densidade potência de equipamentos foi igual a $1,5 \mathrm{~W} / \mathrm{m}^{2}$ na sala durante todas as horas do dia, conforme o RTQ-R (INMETRO, 2012).

Para as temperaturas do solo, considerou-se os valores mensais do próprio arquivo climático. A ventilação natural foi simulada com o modelo do AirFlowNetwork do programa EnergyPlus, que considera as características das aberturas (coeficiente de descarga, expoente de fluxo de ar, infiltração de ar), os coeficientes de pressão do vento, e padrão de utilização das aberturas. Esses parâmetros de ventilação foram configurados de acordo com o RTQ-R (INMETRO, 2012).

Figura 3 - Taxa de ocupação adotada nas simulações.

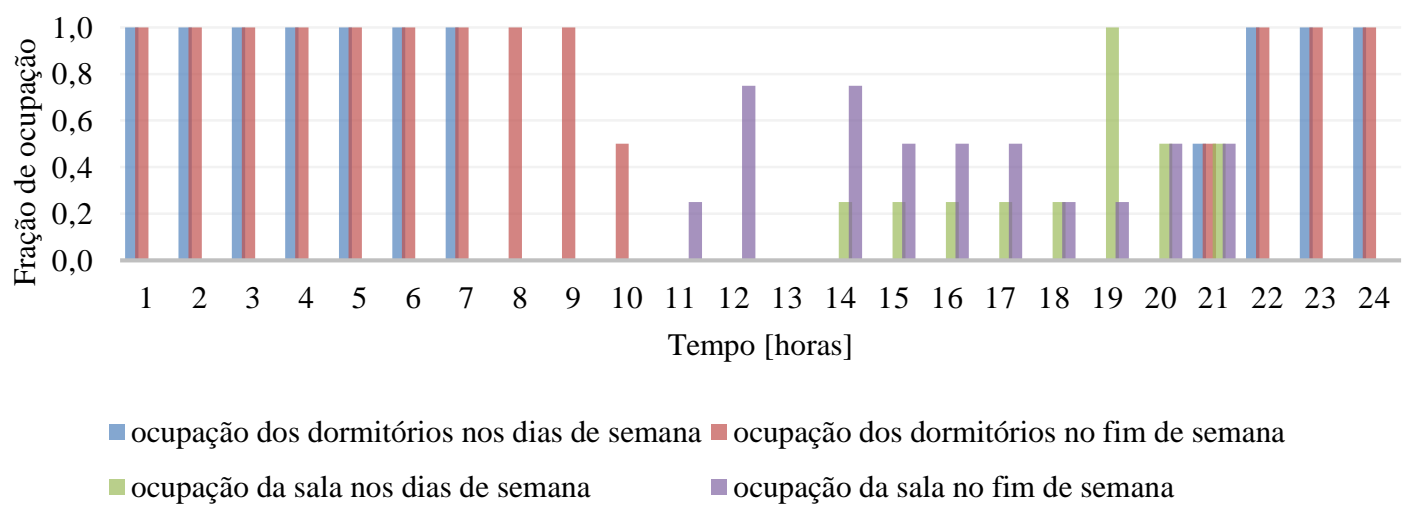

Fonte: Adaptado de RTQ-R (INMETRO, 2012).

Figura 4 - Fração de potência de iluminação adotada nas simulações.

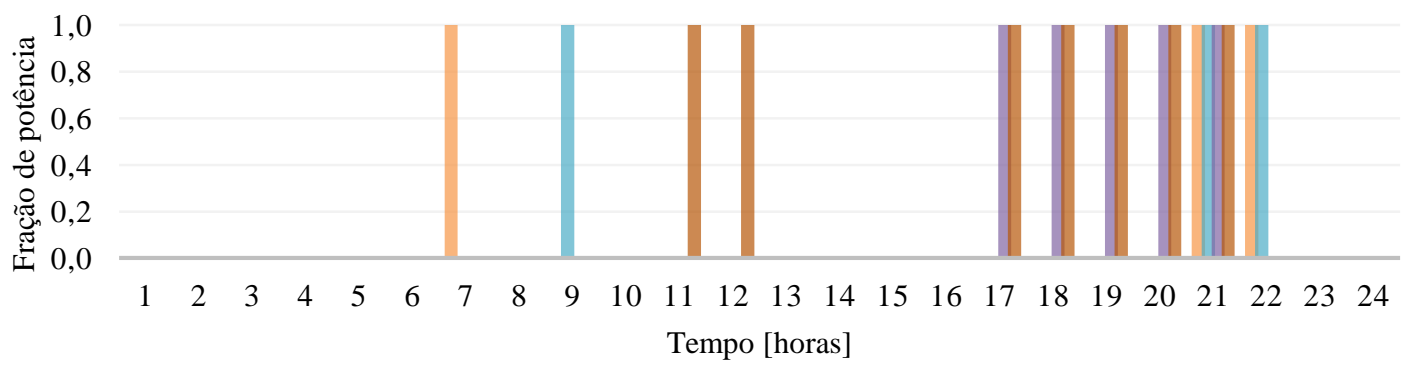

घiluminação nos dormitórios nos dias de semana $\llbracket$ iluminação nos dormitórios no final de semana

घiluminação na sala nos dias de semana \#iluminação na sala no final de semana

Fonte: Adaptado de RTQ-R (INMETRO, 2012). 


\subsection{Projeto de experimento e análise de variância}

O projeto de experimento é uma forma de análise estatística que utiliza a análise de variância em uma amostra multivariada de parâmetros (MONTGOMERY; RUNGER 2012). Essa amostra multivariada é uma combinação fatorial de cada nível de cada parâmetro entre si. O tamanho total da amostra é dado pela Equação (1).

$$
\text { Amostra }=a \times b \ldots \times n
$$

no qual, $\quad$ a = número de níveis no parâmetro $\mathrm{A}$;

b = número de níveis no parâmetro $\mathrm{B}$;

$\mathrm{n} \quad=$ número de níveis no parâmetro $\mathrm{N}$.

A análise de variância foi usada neste trabalho para determinar quais parâmetros podem ser considerados influentes ou irrelevantes em um experimento numérico. O modelo adequado para este tipo de análise estatística precisa ter a possibilidade de ser submetido a diferentes condicionantes e perturbações, de forma a se analisar o impacto de cada uma delas na variável dependente. A simulação computacional possibilita esse tipo de experimento numérico.

Sabe-se que a simulação computacional necessita de inúmeros parâmetros e configurações para realizar a estimativa da variável escolhida. Ao considerar a variação de dois parâmetros A e B em uma simulação computacional, analisando uma variável dependente Y, pode-se calcular a soma dos quadrados conforme as Equações (2) a (5).

$$
\begin{gathered}
S Q(A)=b \times \sum_{i}\left(\bar{y}_{i .}-\bar{y}_{. .}\right)^{2} \\
S Q(B)=a \times \sum_{j}\left(\bar{y}_{. j}-\bar{y}_{. .}\right)^{2} \\
S Q(\text { Total })=\sum_{i} \sum_{j}\left(y_{i j}-\bar{y}_{. .}\right)^{2} \\
S Q(A B)=S Q(\text { Total })-S Q(\text { Erro })-S Q(A)-S Q(B)
\end{gathered}
$$

no qual, a = número de níveis no parâmetro $\mathrm{A}$;

$\mathrm{b} \quad=$ número de níveis no parâmetro $\mathrm{B}$;

$\bar{y}_{i .} \quad=$ média do iésimo nível do parâmetro A;

$\bar{y}_{. .} \quad=$ média de todas as observações;

$\bar{y}_{. j} \quad=$ média o jésimo nível do parâmetro B;

$y_{i j} \quad=$ cada observação individual do iésimo nível do parâmetro A, e do jésimo nível do parâmetro B;

$S Q(A)=$ soma dos quadrados do parâmetro A;

$S Q(B) \quad=$ soma dos quadrados do parâmetro $\mathrm{B}$;

$S Q($ Total $)=$ soma dos quadrados total;

$S Q(A B)=$ soma dos quadrados da interação entre parâmetros;

$S Q($ Erro $) \quad=$ soma dos quadrados do erro.

Calcula-se, com esses dados, a média dos quadrados com a Equação (6) e o valor-F com a Equação (7). O valor-F é a medida de sensibilidade do parâmetro, e pode ser utilizado para comparar quantitativamente diferentes parâmetros.

$$
M Q(A)=\frac{S Q(A)}{a-1}
$$




$$
F(A)=\frac{M Q(A)}{M Q(\text { Erro })}
$$

no qual, $\quad M Q(A) \quad=$ é a média dos quadrados do parâmetro A (analogamente para o parâmetro $\mathrm{B})$;

$F(A)=$ é o valor-F do parâmetro $\mathrm{A}$ (analogamente para $\mathrm{o}$ parâmetro B).

\subsection{Variáveis dependentes do experimento}

O experimento conduzido neste trabalho considera como variável dependente a temperatura operativa de cada ambiente da habitação. Essa temperatura foi considerada como a média aritmética da temperatura do ar e da temperatura média radiante para os fins deste trabalho.

A partir da temperatura operativa horária em cada ambiente da habitação, calculou-se duas variáveis que representam o desempenho térmico da habitação ao longo de um ano: os graus-hora de aquecimento e os graus-hora de resfriamento, como mostram as Equações (8) e (9). Para resumir a análise, uma ponderação dos graus-hora de acordo com a área de cada ambiente foi realizada, encontrando-se um valor comparável de resfriamento e outro de aquecimento para a habitação como um todo.

Ao manter constantes as temperaturas base de $19^{\circ} \mathrm{C}$ para o aquecimento e $26^{\circ} \mathrm{C}$ para o resfriamento, pode-se comparar, de forma mais eficaz, o desempenho de modelos com propriedades termofísicas diferentes.

$$
\begin{aligned}
G H A & =\sum_{i=1}^{8760} s e\left\{\begin{array}{c}
T_{i}<19 \mid 19-T_{i} \\
T_{i}>19 \mid 0
\end{array}\right. \\
G H R & =\sum_{i=1}^{8760} s e\left\{\begin{array}{c}
T_{i}>26 \mid T_{i}-26 \\
T_{i}<26 \mid 0
\end{array}\right.
\end{aligned}
$$

no qual, $\quad G H A=$ são os graus-hora de aquecimento $\left[{ }^{\circ} \mathrm{Ch}\right] ;$

GHR = são os graus-hora de resfriamento $\left[{ }^{\circ} \mathrm{Ch}\right] ;$

$T_{i} \quad=$ são as temperaturas operativas de cada ambiente da habitação em cada hora do ano $\left[{ }^{\circ} \mathrm{C}\right]$;

$i=$ é cada hora do ano [horas].

\subsection{Experimento computacional}

Este item explica o experimento conduzido neste trabalho. O modelo base do Item 2.1 foi configurado para conter a variação de nove parâmetros relacionados às propriedades termofísicas do envelope mostradas na Tabela 1. Esses parâmetros foram variados em dois níveis (mínimo e máximo) por meio de uma combinação fatorial completa, totalizando 512 simulações computacionais. A variação foi realizada considerando componentes construtivos fictícios de uma camada e espessura fixa, alterando-se o valor da condutividade térmica e da massa específica para a obtenção dos valores desejados de transmitância e capacidade térmicas. 
Tabela 1 - Parâmetros variados no experimento.

\begin{tabular}{|c|c|c|c|c|}
\hline Parâmetro & Símbolo & Unidade & Nível 1 & Nível 2 \\
\hline Transmitância térmica das paredes internas & Uparint & {$\left[\mathrm{W} / \mathrm{m}^{2} \mathrm{~K}\right]$} & 1,0 & 3,7 \\
\hline Capacidade térmica das paredes internas & Ctparint & {$\left[\mathrm{kJ} / \mathrm{m}^{2} \mathrm{~K}\right]$} & 49,9 & 250,1 \\
\hline Transmitância térmica das paredes externas & Uparext & {$\left[\mathrm{W} / \mathrm{m}^{2} \mathrm{~K}\right]$} & 1,0 & 3,7 \\
\hline Capacidade térmica das paredes externas & Ctparext & {$\left[\mathrm{kJ} / \mathrm{m}^{2} \mathrm{~K}\right]$} & 49,9 & 250,1 \\
\hline Absortância solar das paredes externas & apar & - & 0,15 & 0,85 \\
\hline Emissividade a onda longa da cobertura & $\varepsilon \mathrm{cob}$ & - & 0,10 & 0,90 \\
\hline Transmitância térmica da cobertura & Ucob & {$\left[\mathrm{W} / \mathrm{m}^{2} \mathrm{~K}\right]$} & 1,0 & 4,0 \\
\hline Capacidade térmica da cobertura & Ctcob & {$\left[\mathrm{kJ} / \mathrm{m}^{2} \mathrm{~K}\right]$} & 20,0 & 200,0 \\
\hline Absortância solar da cobertura & $\alpha \operatorname{cob}$ & - & 0,15 & 0,85 \\
\hline
\end{tabular}

\subsection{Tratamento de dados}

O tratamento dos dados contemplou a análise de variância através do cálculo do valor-F de cada termo envolvido no experimento com a Equação (7) nos graus-hora de aquecimento e resfriamento. A palavra "termo" se refere a cada parâmetro individual ou interação de segunda ordem entre parâmetros. Essa análise foi realizada com o auxílio do programa Minitab 16 (2013).

Determinou-se, também, a distribuição de probabilidades dos graus-hora de aquecimento e resfriamento e separou-se graficamente os dados de acordo com os parâmetros mais influentes detectados, para melhorar a interpretação dos casos obtidos.

\section{RESULTADOS}

\subsection{Análise das variáveis dependentes}

A Figura 5 mostra a distribuição de probabilidades em frequência absoluta para os graushora de resfriamento e aquecimento, considerando os graus-hora médios ponderados pela área útil de cada ambiente da habitação (ou seja, um valor de graus-hora para toda a habitação). Percebe-se claramente a preponderância dos graus-hora de resfriamento para o desempenho térmico dessa habitação para o clima de Campo Grande-MS. O valor máximo obtido para os graus-hora de aquecimento foi de $2024^{\circ} \mathrm{Ch}$, enquanto para os graus-hora de resfriamento foi de $21136^{\circ} \mathrm{Ch}$.

\section{Figura 5 - Frequência absoluta de ocorrência dos graus-hora de resfriamento e} aquecimento resultante das 512 simulações.

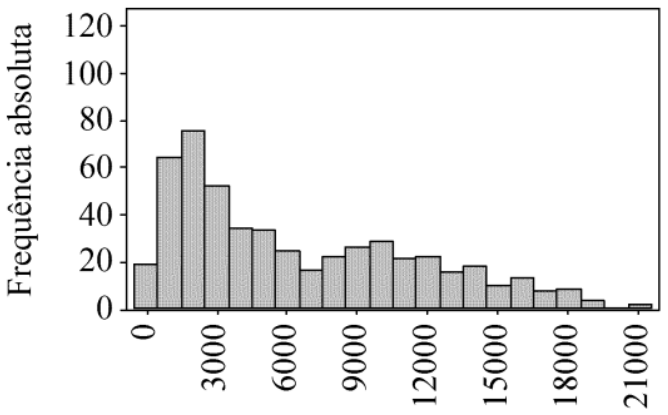

Graus-hora de resfriamento $\left[{ }^{\circ} \mathrm{Ch}\right]$

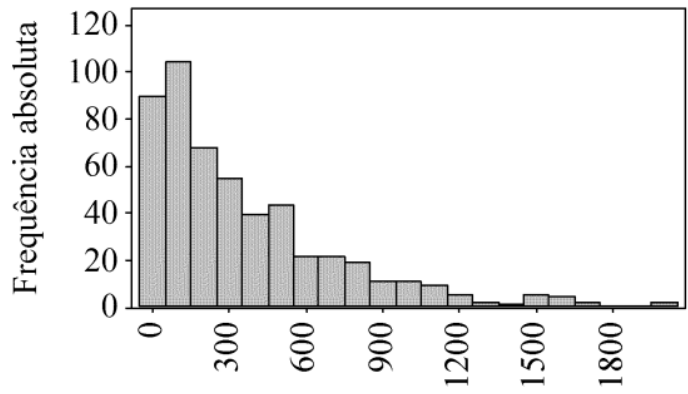

Graus-hora de aquecimento $\left[{ }^{\circ} \mathrm{Ch}\right]$

Também percebe-se que a maior probabilidade de ocorrência dos graus-hora de aquecimento é inferior a $300^{\circ} \mathrm{Ch}$, indicando que apenas algumas combinações específicas 
geraram valores elevados de necessidade de aquecimento ambiental. Nos graus-hora de resfriamento, a maior probabilidade de ocorrência é verificada em valores inferiores a $3000^{\circ} \mathrm{Ch}$, mas há certa probabilidade de ocorrência em valores até $12000^{\circ} \mathrm{Ch}$.

\subsection{Análise de variância}

A Tabela 2 mostra a influência dos termos (parâmetros ou efeitos de segunda ordem) nas variáveis dependentes de graus-hora de aquecimento e resfriamento. Percebe-se que diferentes termos são influentes em cada uma das variáveis. No caso do resfriamento, a absortância solar da cobertura tem quase o dobro da influência do que a transmitância térmica da mesma. E adicionalmente, o efeito de segunda ordem entre esses dois parâmetros (o termo Ucob* $\alpha c o b$ ) é o quarto mais influente no resfriamento.

Análises como estas indicam qual é o parâmetro alvo para se promover estratégias de desempenho térmico.

Para o aquecimento, o parâmetro mais influente é a transmitância térmica das paredes externas e a emissividade da cobertura. No entanto, como mostra a Figura 5, os graushora de aquecimento são relativamente menores do que o de resfriamento. Desta forma, um dimensionamento correto da absortância solar da cobertura e das paredes, e da transmitância térmica da cobertura seriam boas estratégias para aprimorar o desempenho da habitação (considerar valores baixos nesses parâmetros).

Tabela 2 - Influência dos termos nas variáveis dependentes.

\begin{tabular}{|c|c|c|c|}
\hline \multicolumn{2}{|c|}{ Graus-hora de aquecimento } & \multicolumn{2}{|c|}{ Graus-hora de resfriamento } \\
\hline Termo & Valor-F & Termo & Valor-F \\
\hline Uparext & 2216 & $\alpha c o b$ & 8029 \\
\hline$\varepsilon \mathrm{cob}$ & 1855 & Ucob & 4496 \\
\hline Ucob & 1723 & apar & 2964 \\
\hline Ctparext & 1515 & $\mathrm{Ucob}^{*} \alpha \mathrm{cob}$ & 2593 \\
\hline$\varepsilon c o b * U c o b$ & 1062 & Uparext & 1692 \\
\hline$\alpha c o b$ & 609 & Uparext*apar & 913 \\
\hline Ctparint & 586 & Ctparext & 868 \\
\hline apar & 520 & Ctcob & 479 \\
\hline Ctcob & 499 & $\varepsilon \mathrm{cob}^{*} \mathrm{Ucob}$ & 244 \\
\hline
\end{tabular}

No qual: Uparint: Transmitância térmica das paredes internas; CTparint: Capacidade térmica das paredes internas; Uparext: Transmitância térmica das paredes externas; CTparext: Capacidade térmica das paredes externas; $\varepsilon$ cob: Emissividade a onda longa da cobertura; Ucob: Transmitância térmica da cobertura; CTcob: Capacidade térmica da cobertura; $\alpha$ cob: Absortância solar da cobertura; apar: Absortância solar das paredes externas.

Com o auxílio da Figura 6 pode-se perceber visualmente a influência do efeito de segunda ordem detectado para os graus-hora de resfriamento. Quando a absortância solar da cobertura é baixa $(0,15)$, os valores com $1,0 \mathrm{~W} / \mathrm{m}^{2} \mathrm{~K}$ de transmitância térmica da cobertura se diferem pouco dos valores com $4,0 \mathrm{~W} / \mathrm{m}^{2} \mathrm{~K}$. No entanto, se a mesma variação na transmitância térmica ocorre quando a absortância solar da cobertura é alta, grande distância nos dados se observa.

Percebe-se que a transmitância térmica das paredes externas influencia muito mais quando a absortância solar da cobertura é baixa $(0,15)$ e quando a absortância solar das paredes é alta $(0,85)$. Quando se verifica a diferença nos dados com $3,7 \mathrm{~W} / \mathrm{m}^{2} \mathrm{~K}$ de transmitância térmica das paredes externas, em relação aos valores com $1,0 \mathrm{~W} / \mathrm{m}^{2} \mathrm{~K}$, a distância é muito maior no caso de alta absortância solar das paredes. 
Alguns blocos de valores podem ser observados, sendo que os valores mais altos são alcançados no bloco de alta absortância solar e transmitância térmica da cobertura. Percebe-se, também, que os grupos de resultados com alta absortância solar da cobertura $(0,85)$ apresentam dispersão maior nos dados em comparação com os dados de baixa absortância $(0,15)$.

\section{Figura 6 - Análise dos resultados das simulações pelos parâmetros mais influentes nos graus-hora de resfriamento para as 512 simulações.}

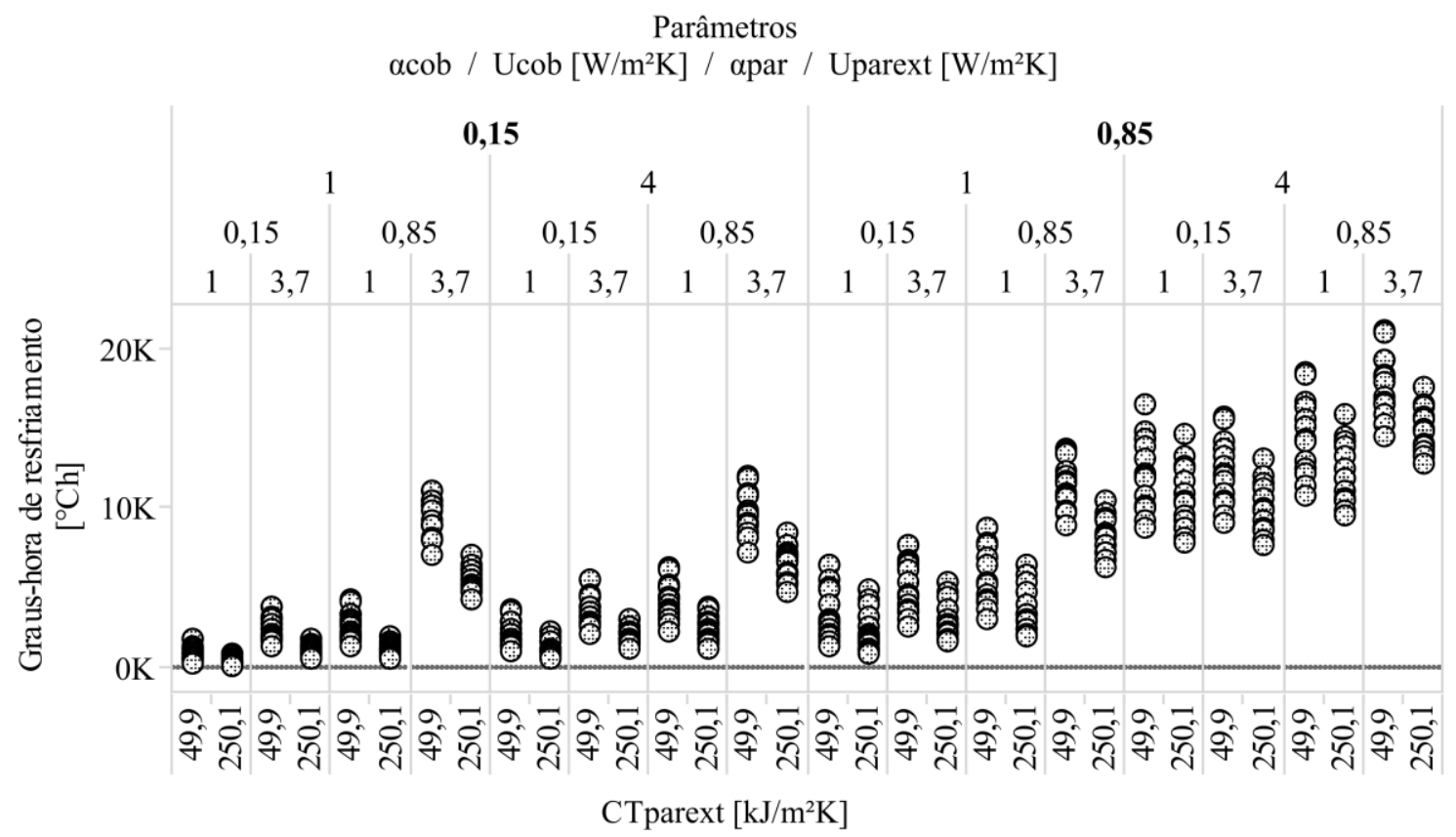

\section{CONCLUSÕES}

O trabalho mostrou uma análise de variância dos parâmetros termofísicos da envoltória de uma habitação unifamiliar no seu desempenho térmico, por meio de projeto de experimento fatorial.

Percebeu-se a importância desse tipo de análise, a qual pode apontar a influência de parâmetros no desempenho térmico das edificações, e também a determinação de quais combinações geraram resultados extremos ou de grande probabilidade de ocorrência.

A análise de variância pode ser aplicada a outros tipos de variáveis dependentes, como consumo de energia ou variáveis de conforto térmico, além de outros tipos de edificações (comerciais, residenciais multifamiliares). A limitação dessa análise é a quantidade de simulações e o esforço computacional para efetuá-las, os quais dependem exponencialmente do número de parâmetros considerados, do nível de variação dos parâmetros, e das características e detalhamentos do modelo computacional adotado.

No caso deste trabalho para o clima específico considerado, com 512 simulações pode-se obter os parâmetros mais influentes (absortância solar e transmitância térmica da cobertura) e os grupos de configurações de parâmetros que poderiam gerar bom desempenho térmico, e as combinações extremas de valores que devem ser evitadas por gerarem desempenho ruim.

\section{AGRADECIMENTOS}

À CAPES, ao CNPq e à FINEP pelo apoio recebido. 


\section{REFERENCIAS}

BROHUS, H.; HEISELBERG, P.; SIMONSEN, A.; SØRENSEN, K. C. Uncertainty of energy consumption assessment of domestic buildings. Building Simulation 2009. Proceedings... p.1022-1029, 2009. Glasgow, Scotland.

CHLELA, F.; HUSAUNNDEE, A.; INARD, C.; RIEDERER, P. A new methodology for the design of low energy buildings. Energy and Buildings, v. 41, n. 9, p. 982-990, 2009.

DOE. US Department of Energy. Weather Data. 2014a. Disponível em <

http://apps1.eere.energy.gov/buildings/energyplus/weatherdata about.cfm $>$ Acesso em 02/04/2014.

DOE. US Department of Energy. EnergyPlus Energy Simulation Software. 2014b.

Disponível em < http://apps1.eere.energy.gov/buildings/energyplus/ > Acesso em 02/04/2014.

HOPFE, C. J.; HENSEN, J. L. M. Uncertainty analysis in building performance simulation for design support. Energy and Buildings, v. 43, n. 10, p. 2798-2805, 2011.

INMETRO. Ministério do desenvolvimento, indústria e comércio. Regulamento técnico da qualidade para o nível de eficiência energética edificações residenciais. RTQ-R. Portaria n.18, de 16 de Janeiro de 2012. INMETRO, 2012.

ISO International Organization for Standardization. ISO 13790 - Energy performance of buildings - calculation of energy use for space heating and cooling. Genebra, 2008.

MARA, T. A.; TARANTOLA, S. Application of global sensitivity analysis of model output to building thermal simulations. Building Simulation, v. 1, n. 4, p. 290-302, 2008.

MECHRI, H. E.; CAPOZZOLI, A.; CORRADO, V. USE of the ANOVA approach for sensitive building energy design. Applied Energy, v. 87, n. 10, p. 3073-3083, 2010.

MINITAB. Minitab 16 Software. 2013. Disponível em < http://www.minitab.com/ > Acesso em 02/04/2014.

MONTGOMERY, D. C.; RUNGER, C. Estatística aplicada e probabilidade para engenheiros. John Wiley \& Sons, Inc. Edição LTC. Rio de Janeiro, 2012.

SALTELLI, A.; TARANTOLA, S.; CAMPOLONGO, F. Sensitivity Analysis as an Ingredient of Modeling. Statistical Science, v. 15, n. 4, p. 377-395, 2000.

SANCHEZ, D.G.; LACARRIÈRE, B.; MUSY, M.; BOURGES, B. Application of sensitivity analysis in building energy simulations: Combining first- and second-order elementary effects methods. Energy and Buildings, v. 68, p. 741-750, 2014.

TIAN, W. A review of sensitivity analysis methods in building energy analysis. Renewable and Sustainable Energy Reviews, v. 20, p. 411-419, 2013. 